\title{
APPROXIMATE SOLUTIONS OF EQUATIONS DEFINED BY COMPLEX SPHERICAL MULTIPLIER OPERATORS
}

\author{
C. P. OLIVEIRA
}

Received 20 January 2004 and in revised form 9 December 2004

This paper studies, in a partial but concise manner, approximate solutions of equations defined by complex spherical multiplier operators. The approximations are from native spaces embedded in Sobolev-type spaces and derived from the use of positive definite functions to perform spherical interpolation.

\section{Introduction}

Let $\Omega_{2 q}$ be the unit sphere in $\mathbb{C}^{q}, q \geq 2$. If the usual inner product between $z$ and $w$ in $\mathbb{C}^{q}$ is denoted by $\langle z, w\rangle$, then

$$
\Omega_{2 q}=\left\{z \in \mathbb{C}^{q}:\langle z, z\rangle=1\right\} .
$$

This paper is concerned with approximate solutions of an equation of the form

$$
T(f)=g,
$$

in which $T: L^{2}\left(\Omega_{2 q}\right) \rightarrow L^{2}\left(\Omega_{2 q}\right)$ is a spherical multiplier operator. Ideally, the domain $D_{T}$ of $T$ should contain complex spherical harmonics up to a certain degree. The term harmonic refers to a function of $q$ complex variables belonging to the kernel of the Laplacian

$$
\Delta_{(2 q)}:=4 \sum_{j=1}^{q} \frac{\partial^{2}}{\partial z_{j} \partial \bar{z}_{j}}, \quad z \in \mathbb{C}^{q} .
$$

A complex spherical harmonic is then the restriction to $\Omega_{2 q}$ of a harmonic polynomial of bidegree $(m, n)$. A polynomial has bidegree $(m, n)$ when it is homogeneous of degree $m$ with respect to $z \in \mathbb{C}^{q}$ and homogeneous of degree $n$ with respect to $\bar{z}$.

To better explain what a multiplier operator is in our context, we need to introduce some notation. We will write

$$
\left\{Y_{m, n}^{j}: j=1, \ldots, d(m, n)\right\}
$$


to denote a fixed orthonormal basis for $\mathscr{H}_{m, n}\left(\Omega_{2 q}\right)$, the space of complex spherical harmonics of bidegree $(m, n)$. Orthonormality refers to the usual inner product $\langle\cdot, \cdot\rangle_{2}$ of $L^{2}\left(\Omega_{2 q}\right)$, that is,

$$
\langle f, g\rangle_{2}:=\int_{\Omega_{2 q}} f(z) \overline{g(z)} d \sigma_{q}(z), \quad f, g \in L^{2}\left(\Omega_{2 q}\right)
$$

in which $d \sigma_{q}$ denotes the Lebesgue measure over $\Omega_{2 q}$ so normalized that

$$
\int_{\Omega_{2 q}} d \sigma_{q}(z):=\omega_{q}
$$

the surface measure of $\Omega_{2 q}$.

Every function $f$ of $L^{2}\left(\Omega_{2 q}\right)$ has a Fourier-type expansion in the form

$$
f \sim \sum_{m, n \in \mathbb{Z}_{+}} \sum_{j=1}^{d(m, n)} \hat{f}_{m, n}(j) Y_{m, n}^{j}, \quad \hat{f}_{m, n}(j):=\left\langle f, Y_{m, n}^{j}\right\rangle_{2} .
$$

The condensed expansion of $f$ is given by

$$
f \sim \sum_{m, n \in \mathbb{Z}_{+}} s_{m, n}(f), \quad s_{m, n}(f):=\sum_{j=1}^{d(m, n)} \hat{f}_{m, n}(j) Y_{m, n}^{j} .
$$

A complex spherical multiplier operator $T$ is then an operator

$$
T: L^{2}\left(\Omega_{2 q}\right) \longrightarrow L^{2}\left(\Omega_{2 q}\right)
$$

characterized by the following property: there exists a double-indexed sequence $\left\{c_{m, n}\right\}_{m, n \in \mathbb{Z}_{+}}$of complex numbers, the spherical symbol, such that $c_{m, n}=\bar{c}_{n, m}, m, n \in \mathbb{Z}_{+}$, and

$$
\widehat{T(f)}_{m, n}(j)=c_{m, n} \hat{f}_{m, n}(j), \quad f \in L^{2}\left(\Omega_{2 q}\right), j=1, \ldots, d(m, n), m, n \in \mathbb{Z}_{+} .
$$

In particular, if $T$ is a spherical multiplier operator, then

$$
T\left(Y_{m, n}^{j}\right)=c_{m, n} Y_{m, n}^{j}, \quad j=1, \ldots, d(m, n), m, n \in \mathbb{Z}_{+} .
$$

Henceforth, unless stated otherwise, the letter $T$ will denote a spherical multiplier operator associated with spherical symbol $\left\{c_{m, n}\right\}_{m, n \in \mathbb{Z}_{+}}$.

The approximations in this paper will take place in spaces constructed from a fixed basis function $K:\{z \in \mathbb{C}:|z| \leq 1\} \mapsto \mathbb{C}$ having an expansion in the form (see [9])

$$
K(z)=\sum_{m, n \in \mathbb{Z}_{+}} a_{m, n}^{q}(K) P_{m, n}^{q-2}(z), \quad a_{m, n}^{q}(K) \geq 0, K(1)<\infty .
$$

The function $P_{m, n}^{q-2}$ represents the disk polynomial given by

$$
P_{m, n}^{q-2}\left(r e^{i \theta}\right)=r^{|m-n|} e^{i(n-m) \theta} P_{m \wedge n}^{(q-2,|m-n|)}\left(2 r^{2}-1\right), \quad z=r e^{i \theta},
$$


where $P_{m \wedge n}^{(q-2,|m-n|)}$ is the Jacobi polynomial of degree $m \wedge n$ associated with the pair $(q-2$, $|m-n|)$ and so normalized that $P_{m \wedge n}^{(q-2,|m-n|)}(1)=1$. Details about disk polynomials can be found in $[1,4]$. For now, we mention the addition formula for disk polynomials

$$
P_{m, n}^{q-2}(\langle z, w\rangle)=\frac{\omega_{q}}{d(m, n)} \sum_{j=1}^{d(m, n)} Y_{m, n}^{j}(z) \overline{Y_{m, n}^{j}(w)}, \quad z, w \in \Omega_{2 q} .
$$

The basis function $K$ is assumed to be smooth with respect to $T$ in the following sense:

$$
\sum_{m, n \in \mathbb{Z}_{+}} a_{m, n}^{q}(K)\left|c_{m, n}\right|^{2}<\infty
$$

Among other things, such condition allows us to use $K$ to construct spaces where (1.2) makes sense and has solutions. In addition, we have the basis function given by

$$
K_{T}(z):=\sum_{m, n \in \mathbb{Z}_{+}} a_{m, n}^{q}(K)\left|c_{m, n}\right|^{2} P_{m, n}^{q-2}(z), \quad a_{m, n}^{q}(K)\left|c_{m, n}\right|^{2} \geq 0, K_{T}(1)<\infty,
$$

to be used in Section 3.

We will approximate the solutions $f$ of (1.2) by functions of the form

$$
s_{f}=\sum_{j=1}^{N} c_{j}\left(\delta_{j} \circ T^{w}\right) K(\langle\cdot, w\rangle), \quad w \in \Omega_{2 q}, c_{j} \in \mathbb{C} .
$$

In this equation,

$$
\delta_{j}(h):=h\left(w_{j}\right), \quad h \in L^{2}\left(\Omega_{2 q}\right), j=1, \ldots, N,
$$

where the points $w_{1}, \ldots, w_{N}$ are distinct over $\Omega_{2 q}$. The notation $\left(\delta_{j} \circ T^{w}\right) K(\langle\cdot, w\rangle)$ means that $\delta_{j} \circ T$ is acting on the function

$$
w \in \Omega_{2 q} \longmapsto K(\langle\cdot, w\rangle) .
$$

If $\left\{\delta_{1}, \ldots, \delta_{N}\right\}$ is linearly independent and $f$ is in an appropriate space, then we will show that $s_{f}$ is the unique function in the space

$$
\operatorname{span}\left\{\left(\delta_{j} \circ T^{w}\right) K(\langle\cdot, w\rangle): j=1, \ldots, N\right\}
$$

that is a solution of the interpolation

$$
\left(\delta_{j} \circ T\right)(w)=\left(\delta_{j} \circ T\right)(f), \quad j=1, \ldots, N .
$$

Assuming additional smoothness conditions on $T$ and on the points $w_{1}, \ldots, w_{N}$, we will establish error estimates for the approximation of solutions of (1.2) by $s_{f}$ in the norm of spaces of Sobolev-type in which the native spaces are continuously embedded.

We would like to observe that the approach taken here is known (see $[3,5,11])$. What makes a difference here is that our paper refines and details some ideas presented in other sources, putting them into the more general complex setting. In addition, estimates not considered until now are investigated. 


\section{Native spaces}

In this section, we briefly discuss native spaces associated with sequences and functions. They are Hilbert spaces where the sequences and functions act as a generalized reproducing kernel. For instance, we will see in Section 3 that a spherical multiplier operator is surjective when its domain and image are appropriate native spaces in $L^{2}\left(\Omega_{2 q}\right)$. Native spaces in the real setting were considered in $[6,7]$.

The native space associated with a complex sequence $\beta:=\left\{b_{m, n}\right\}_{m, n \in \mathbb{Z}_{+}}$is

$$
\mathcal{N}_{\beta}:=\left\{f \in L^{2}\left(\Omega_{2 q}\right):\|f\|_{\beta}<\infty, s_{k, l}(f)=0,(k, l) \notin A_{\beta}\right\},
$$

in which

$$
\begin{gathered}
A_{\beta}:=\left\{(k, l) \in \mathbb{Z}_{+}^{2}: b_{k, l} \neq 0\right\}, \\
\|f\|_{\beta}^{2}:=\frac{1}{\omega_{q}} \sum_{(m, n) \in A_{\beta}} \frac{d(m, n)}{\left|b_{m, n}\right|}\left\|s_{m, n}(f)\right\|_{2}^{2} .
\end{gathered}
$$

It is very easy to see that $\|\cdot\|_{\beta}$ is a norm obtainable from the inner product

$$
\langle f, g\rangle_{\beta}:=\frac{1}{\omega_{q}} \sum_{(m, n) \in A_{\beta}} \frac{d(m, n)}{\left|b_{m, n}\right|} \sum_{j=1}^{d(m, n)} \hat{f}_{m, n}(j) \overline{\hat{g}_{m, n}(j)}, \quad f, g \in \mathcal{N}_{\beta}
$$

In addition, we have the following result.

Theorem 2.1. The space $\mathcal{N}_{\beta}$ possesses these properties:

(i) $\mathscr{H}_{m, n}\left(\Omega_{2 q}\right) \subseteq \mathcal{N}_{\beta}$ if and only if $(m, n) \in A_{\beta}$;

(ii) $\left(\mathcal{N}_{\beta},\langle\cdot, \cdot\rangle_{\beta}\right)$ is a Hilbert space;

(iii) the space $\operatorname{span}\left\{Y_{m, n}^{j}: j=1, \ldots, d(m, n),(m, n) \in A_{\beta}\right\}$ is dense in $\mathcal{N}_{\beta}$.

Proof. (i) If $(m, n) \in A_{\beta}$, then

$$
s_{k, l}\left(Y_{m, n}^{j}\right)=\left\{\begin{array}{ll}
Y_{m, n}^{j}, & (k, l)=(m, n), \\
0, & (k, l) \neq(m, n),
\end{array} \quad j=1, \ldots, d(m, n) .\right.
$$

Hence,

$$
\left\|Y_{m, n}^{j}\right\|_{\beta}^{2}=\frac{d(m, n)}{\omega_{q}\left|b_{m, n}\right|}, \quad s_{k, l}\left(Y_{m, n}^{j}\right)=0,(k, l) \notin A_{\beta} .
$$

Thus, $\mathscr{H}_{m, n}\left(\Omega_{2 q}\right) \subset \mathcal{N}_{\beta}$. The converse is immediate.

(ii) If $\langle f, f\rangle_{\beta}=0$ for some $f \in \mathcal{N}_{\beta}$, then $s_{m, n}(f)=0,(m, n) \in A_{\beta}$. Since $s_{m, n}(f)=0$, $(m, n) \notin A_{\beta}$, by definition, it follows that $s_{m, n}(f)=0$ for all $m$ and $n$. The linear independence of the set $\left\{Y_{m, n}^{j}: j=1, \ldots, d(m, n), m, n \in \mathbb{Z}_{+}\right\}$now implies that

$$
\widehat{f}_{m, n}(j)=0, \quad j=1, \ldots, d(m, n), m, n \in \mathbb{Z}_{+},
$$


that is, $f=0$. The other properties needed here are standard and require the following two facts: $\left(L^{2}\left(\Omega_{2 q}\right),\langle\cdot, \cdot\rangle_{2}\right)$ is a Hilbert space and the mapping $f \in L^{2}\left(\Omega_{2 q}\right) \mapsto s_{m, n}(f) \in$ $L^{2}\left(\Omega_{2 q}\right)$ is a linear operator.

(iii) It suffices to use (ii) and a known result on total sets in Hilbert spaces (see [4, page 169]).

Native spaces are continuously imbedded in $\left(\mathscr{C}\left(\Omega_{2 q}\right),\|\cdot\|_{\infty}\right)$.

Theorem 2.2. If $|\beta|:=\left\{\left|b_{m, n}\right|\right\}_{m, n \in \mathbb{Z}_{+}}$is summable, then $\mathcal{N}_{\beta}$ is a subspace of $\mathscr{C}\left(\Omega_{2 q}\right)$. In addition, there exists a positive constant $C_{0}$, not depending on $f$, such that

$$
\|f\|_{\infty} \leq C_{0}\|f\|_{\beta}, \quad f \in \mathcal{N}_{\beta}
$$

Proof. It suffices to show that the condensed expansion (1.8) of a function $f$ in $\mathcal{N}_{\beta}$ is absolutely convergent. To do that, we use the Cauchy-Schwarz inequality and the addition formula for spherical harmonics to obtain

$$
\begin{aligned}
\left(\sum_{m, n \in \mathbb{Z}_{+}}\left|s_{m, n}(f)(z)\right|\right)^{2} & \left(\sum_{(m, n) \in A_{\beta}}\left|s_{m, n}(f)(z)\right|\right)^{2} \\
& =\sum_{(m, n) \in A_{\beta}} \sum_{j=1}^{d(m, n)} \frac{\left(\left|b_{m, n}\right| \omega_{q}\right)^{1 / 2}}{d(m, n)^{1 / 2}}\left|Y_{m, n}^{j}(z)\right| \frac{d(m, n)^{1 / 2}}{\left(\left|b_{m, n}\right| \omega_{q}\right)^{1 / 2}}\left|\hat{f}_{m, n}(j)\right| \\
& \leq \sum_{(m, n) \in A_{\beta}} \frac{\left|b_{m, n}\right| \omega_{q}}{d(m, n)} \sum_{j=1}^{d(m, n)}\left|Y_{m, n}^{j}(z)\right|^{2} \sum_{(m, n) \in A_{\beta}} \frac{d(m, n)}{\omega_{q}\left|b_{m, n}\right|} \sum_{j=1}^{d(m, n)}\left|\hat{f}_{m, n}(j)\right|^{2} \\
& \leq\left(\sum_{m, n \in \mathbb{Z}_{+}}\left|b_{m, n}\right|\right)\left(\frac{1}{\omega_{q}} \sum_{(m, n) \in A_{\beta}} \frac{d(m, n)}{\left|b_{m, n}\right|} \|\left. s_{m, n}(f)\right|_{2} ^{2}\right)^{2} \\
& =C_{0}^{2}\|f\|_{\beta}^{2}, \quad z \in \Omega_{2 q}, \quad
\end{aligned}
$$

in which $C_{0}^{2}=\sum_{m, n \in \mathbb{Z}_{+}}\left|b_{m, n}\right|<\infty$.

Corollary 2.3. If $|\beta|$ is summable and $z \in \Omega_{2 q}$, then the point-evaluation functional $\delta_{z}$ given by $\delta_{z}(f)=f(z), f \in \mathcal{N}_{\beta}$, is continuous.

\section{Multiplier operators and native spaces}

This section contains technical results to be used later and examples of multiplier operators. In particular, we establish a setting where (1.2) has a unique solution. 
We begin recalling the concept of spherical convolution. If $K$ is as in (1.12) and such that $a_{m, n}^{q}(K)=a_{n, m}^{q}(K)$, then the spherical convolution operator with $K$ is given by

$$
\Psi_{K}(f):=\int_{\Omega_{2 q}} K(\langle z, \cdot\rangle) f(z) d \sigma_{q}(z), \quad f \in L^{2} \Omega_{2 q} .
$$

The addition formula for disk polynomials and the Fubini-Tonelli's theorem (see [2, page 223]) imply that $\Psi_{K}$ is a multiplier operator with spherical symbol

$$
\frac{a_{m, n}^{q}(K) \omega_{q}}{d(m, n)}, \quad m, n \in \mathbb{Z}_{+} .
$$

More information about this operator, including a complex version of the Funk-Hecke formula, can be found in [8].

Next, we explore the native space associated with a function $K$ as in (1.12). When the sequence $\beta$ in the definition of native space is $\left\{a_{m, n}^{q}(K)\right\}_{m, n \in \mathbb{Z}_{+}}$, we write $\mathcal{N}_{K}:=\mathcal{N}_{\beta}$, $\|\cdot\|_{K}:=\|\cdot\|_{\beta}$ and $A_{K}:=A_{\beta}$. An analogous remark applies to basis function $K_{T}$.

THeOREM 3.1. Let $T$ be a multiplier operator and $w$ a fixed element of $\Omega_{2 q}$. If $K$ is a function as in (1.12) and obeying (1.15), then functions

$$
z \in \Omega_{2 q} \longmapsto K(\langle z, w\rangle), \quad z \in \Omega_{2 q} \longmapsto T^{w}(K\langle z, w\rangle), \quad z \in \Omega_{2 q} \longmapsto T^{z}(K\langle z, w\rangle)
$$

belong to $\mathcal{N}_{K}$.

Proof. Write $g(z)=K(\langle z, w\rangle), z \in \Omega_{2 q}$. We obtain

$$
\widehat{g}_{m, n}(j)=\frac{a_{m, n}^{q}(K) \omega_{q}}{d(m, n)} \overline{Y_{m, n}^{j}(w)}, \quad j=1, \ldots, d(m, n), m, n \in \mathbb{Z}_{+} .
$$

The addition formula yields

$$
\begin{aligned}
\left\|s_{m, n}(g)\right\|_{2}^{2} & =\sum_{j=1}^{d(m, n)}\left|\hat{g}_{m, n}(j)\right|^{2}=\frac{a_{m, n}^{q}(K)^{2} \omega_{q}^{2}}{d(m, n)^{2}} \sum_{j=1}^{d(m, n)}\left|Y_{m, n}^{j}(w)\right|^{2} \\
& =\frac{a_{m, n}^{q}(K)^{2} \omega_{q}}{d(m, n)}, \quad m, n \in \mathbb{Z}_{+} .
\end{aligned}
$$

Hence,

$$
\|g\|_{K}^{2}=\sum_{(m, n) \in A_{K}} \frac{d(m, n)}{a_{m, n}^{q}(K) \omega_{q}} \frac{a_{m, n}^{q}(K)^{2} \omega_{q}}{d(m, n)}=\sum_{(m, n) \in A_{K}} a_{m, n}^{q}(K)<\infty .
$$


The proofs in the other two cases are very much alike. We include the details for the function $h(z)=T^{z} K(\langle z, w\rangle), z \in \Omega_{2 q}$. As above

$$
\begin{gathered}
\hat{h}_{m, n}(j)=\frac{a_{m, n}^{q}(K) \omega_{q}}{d(m, n)} c_{m, n} \overline{Y_{m, n}^{j}(w)}, \quad j=1,2, \ldots, d(m, n), m, n \in \mathbb{Z}, \\
\left\|s_{m, n}(h)\right\|_{2}^{2}=\sum_{j=1}^{d(m, n)}\left|\hat{h}_{m, n}(j)\right|^{2}=\frac{a_{m, n}^{q}(K)^{2} \omega_{q}}{d(m, n)}\left|c_{m, n}\right|^{2}, \quad m, n \in \mathbb{Z} .
\end{gathered}
$$

Thus,

$$
\|h\|_{K}^{2}=\sum_{(m, n) \in A_{K}} \frac{d(m, n)}{a_{m, n}^{q}(K) \omega_{q}} \frac{a_{m, n}^{q}(K)^{2} \omega_{q}}{d(m, n)}\left|c_{m, n}\right|^{2}=\sum_{(m, n) \in A_{K}} a_{m, n}^{q}(K)\left|c_{m, n}\right|^{2}<\infty .
$$

Finally, note that if $(k, l) \notin A_{K}$, then $s_{k, l}(g)=0$ and $s_{k, l}(h)=0$.

Theorem 3.2. Let $T$ and $K$ be as in the previous theorem. Then $T^{z} T^{w}(K\langle z, w\rangle)$ defines a uniformly convergent series with sum $K_{T}(\langle z, w\rangle)$.

Proof. For $z, w \in \Omega_{2 q}$, the addition formula implies that

$$
T^{z} T^{w} K(\langle z, w\rangle) \sim \sum_{m, n \in \mathbb{Z}_{+}} a_{m, n}^{q}(K)\left|c_{m, n}\right|^{2} P_{m, n}^{q-2}(\langle z, w\rangle)
$$

Condition (1.15) completes the proof.

Below, we investigate some connections between the native spaces of $K$ and $K_{T}$. In particular, we present a setting in which $T$ becomes surjective, a condition that guarantees the existence of solutions of the equation $T(f)=g$. This seems to be forgotten in the real setting.

Theorem 3.3. Let $T$ be a multiplier operator and $K$ as described in (1.12). Then $\mathcal{N}_{K_{T}} \subseteq$ $T\left(\mathcal{N}_{K}\right)$.

Proof. Let $g \in \mathcal{N}_{K_{T}}$. The function $f \in L^{2}\left(\Omega_{2 q}\right)$ with Fourier coefficients given by

$$
\hat{f}_{m, n}(j)= \begin{cases}\frac{\hat{g}_{m, n}(j)}{c_{m, n}}, & (m, n) \in A_{K_{T}} \\ 0, & (m, n) \notin A_{K_{T}}\end{cases}
$$

satisfies

$$
s_{m, n}(f)= \begin{cases}\frac{s_{m, n}(g)}{c_{m, n}}, & (m, n) \in A_{K_{T}} \\ 0, & (m, n) \notin A_{K_{T}} .\end{cases}
$$


Consequently,

$$
\begin{aligned}
\sum_{(m, n) \in A_{K}} \frac{d(m, n)}{a_{m, n}^{q}(K)}\left\|s_{m, n}(f)\right\|_{2}^{2} & =\sum_{(m, n) \in A_{K_{T}}} \frac{d(m, n)}{a_{m, n}^{q}(K)\left|c_{m, n}\right|^{2}}\left\|s_{m, n}(g)\right\|_{2}^{2} \\
& =\omega_{q}\|g\|_{K_{T}}^{2}<\infty
\end{aligned}
$$

that is, $\|f\|_{K}<\infty$. Since the definition of $f$ reveals that $s_{m, n}(f)=0,(m, n) \notin A_{K}$, it is now clear that $f \in \mathcal{N}_{K}$. Thus, $g=T(f)$. In addition, the Fourier series of $g$ and $T(f)$ coincide in $L^{2}\left(\Omega_{2 q}\right)$.

In some situations, the functions in the space $\mathcal{N}_{K}$ need to have some desirable additional smoothness. Theorem 3.4 below reveals how one can substitute that space by another one composed of smoother functions and not too much different from the original space. It has to do with the composition $\Psi_{K} \circ K(\langle\cdot, w\rangle)$ for some $w \in \Omega_{2 q}$.

Theorem 3.4. Let $K$ and $\Psi_{K}$ be as in Theorem 3.1. If $w \in \Omega_{2 q}$, then the function

$$
z \in \Omega_{2 q} \longmapsto \Psi_{K} \circ K(\langle\cdot, w\rangle)(z)
$$

is of form $K_{1}(\langle\cdot, w\rangle)$, where $K_{1}$ is representable as in (1.12). In addition, $K_{1}$ obeys (1.15) if $K$ does.

Proof. Using the additional formula and the orthogonality of spherical harmonics, we obtain

$$
\begin{aligned}
\Psi_{K}(K(\langle\cdot, w\rangle)(z)) & =\int_{\Omega_{2 q}} K(\langle\zeta, z\rangle) K(\langle\zeta, w\rangle) d \sigma_{q}(\zeta) \\
& =\sum_{m, n \in \mathbb{Z}_{+}} a_{m, n}^{q}(K) \int_{\Omega_{2 q}} K(\langle\zeta, z\rangle) P_{m, n}^{q-2}(\langle\zeta, w\rangle) d \sigma_{q}(\zeta) \\
& =\sum_{m, n \in \mathbb{Z}_{+}} \frac{a_{m, n}^{q}(K) \omega_{q}}{d(m, n)} \sum_{j=1}^{d(m, n)} \Psi_{K}\left(Y_{m, n}^{j}\right)(z) \overline{Y_{m, n}^{j}(w)} \\
& =\sum_{m, n \in \mathbb{Z}_{+}}\left(\frac{a_{m, n}^{q}(K) \omega_{q}}{d(m, n)}\right)^{2} \sum_{j=1}^{d(m, n)} Y_{m, n}^{j}(z) \overline{Y_{m, n}^{j}(w)} \\
& =\sum_{m, n \in \mathbb{Z}_{+}} \frac{a_{m, n}^{q}(K)^{2} \omega_{q}}{d(m, n)} P_{m, n}^{q-2}(\langle z, w\rangle):=K_{1}(\langle z, w\rangle), \quad z \in \Omega_{2 q} .
\end{aligned}
$$

It follows that $K_{1}$ has an expansion as in (1.12) because

$$
\sum_{m, n \in \mathbb{Z}_{+}} \frac{a_{m, n}^{q}(K) \omega_{q}}{d(m, n)}<\infty, \quad \lim _{m, n \rightarrow \infty} a_{m, n}^{q}(K)=0 .
$$

A similar argument resolves the last statement of the theorem.

The function $\Psi_{K}(K(\langle\cdot, w\rangle))$ appearing above is frequently called the spherical convolution of $K$ by itself and it is denoted by $K * K$. Although $A_{K * K}=A_{K}$, the spaces $\mathcal{N}_{K * K}$ and $\mathcal{N}_{K}$ are usually different. 
For future use, we mention the series representation of $K_{T} * K_{T}(\langle\cdot, w\rangle)$ :

$$
K_{T} * K_{T}(\langle\cdot, w\rangle)=\sum_{m, n \in \mathbb{Z}_{+}} \frac{a_{m, n}^{q}(K)^{2} \omega_{q}}{d(m, n)}\left|c_{m, n}\right|^{4} P_{m, n}^{q-2}(\langle\cdot, w\rangle), \quad w \in \Omega_{2 q} .
$$

\section{Native spaces and Sobolev spaces}

We will work with estimates in the spaces $\mathcal{N}_{K}$ and other spaces containing them. Among such spaces are certain Sobolev-type spaces on $\Omega_{2 q}$ which we now define.

Henceforth, $-\lambda_{m, n}$ will denote the sole eigenvalue of the restriction of the LaplaceBeltrami operator to the space $\mathscr{H}_{m, n}\left(\Omega_{2 q}\right)$ while $\lambda$ will denote the double-indexed sequence $\left\{\lambda_{m, n}\right\}_{m, n \in \mathbb{Z}_{+}}$. Given a real number $\mu$ and a real sequence $\alpha:=\left\{\alpha_{m, n}\right\}_{m, n \in \mathbb{Z}_{+}}$satisfying

$$
1+\alpha_{m, n} \leq C_{\alpha}\left(1+\lambda_{m, n}\right), \quad m, n \in \mathbb{Z}_{+}
$$

for some positive constant $C_{\alpha}>0$, the Sobolev-type space associated with $\alpha$ and $\mu$ is the space

$$
\mathscr{H}^{\mu}(\alpha):=\left\{f \in L^{2}\left(\Omega_{2 q}\right):\|f\|_{\alpha, \mu}<\infty\right\}
$$

in which

$$
\|f\|_{\alpha, \mu}^{2}:=\sum_{m, n \in \mathbb{Z}_{+}}\left(1+\alpha_{m, n}\right)^{\mu}\left\|s_{m, n}(f)\right\|_{2}^{2}
$$

Since $\lambda_{m, n}=(m+n)(m+n+2 q-2)$, it is not surprising at all that the following equality of Sobolev-type spaces holds. This explains why some authors use the sequence $\delta$ instead of $\lambda$ in the above definition.

Theorem 4.1. If $\delta:=\{m+n\}_{m, n \in \mathbb{Z}_{+}}$, then $\mathscr{H}^{2 \mu}(\delta)=\mathscr{H}^{\mu}(\lambda), \mu \in \mathbb{R}$.

Proof. Analyzing the graphs of the functions $f_{q}:[0, \infty) \rightarrow \mathbb{R}$ given by

$$
f_{q}(x)=\frac{x^{2}+(2 q-2) x+1}{(1+x)^{2}}, \quad q=2,3, \ldots,
$$

we obtain the inequalities

$$
1 \leq f_{q}(x) \leq \frac{q}{2}
$$

Since $f_{q}(m+n)=\left(1+\lambda_{m, n}\right)(1+m+n)^{-2}, m, n \in \mathbb{Z}_{+}$, it follows that

$$
(1+m+n)^{2} \leq\left(1+\lambda_{m, n}\right) \leq \frac{q}{2}(1+m+n)^{2}, \quad m, n \in \mathbb{Z}_{+} .
$$

Hence, there is a positive constant $K:=K(\mu, q)$ such that

$$
(1+m+n)^{2 \mu} \leq\left(1+\lambda_{m, n}\right)^{\mu} \leq K(1+m+n)^{2 \mu}, \quad \mu \in \mathbb{R} .
$$

The assertion of the theorem follows. 
Next, we will consider spherical multiplier operators possessing polynomial decay. For such operators, it is possible to visualize a Sobolev-type space setting where (1.2) makes sense and has solutions. This additional assumption on the operators is not as inconvenient as it seems because all important examples considered here and elsewhere have some decay.

A spherical multiplier operator $T$ is pseudodifferential of order $v \in \mathbb{R}$ if there exist positive constants $c$ and $C$ such that

$$
c\left(1+\lambda_{m, n}\right)^{\nu} \leq\left|c_{m, n}\right|^{2} \leq C\left(1+\lambda_{m, n}\right)^{\nu}, \quad m, n \in \mathbb{Z}_{+}
$$

Theorem 4.2. Let $K$ be a function as in (1.12) and obeying (1.15). If $T$ is pseudo-differential of order $v$ then $\mathcal{N}_{K} \subseteq \mathscr{H}^{v}(\lambda)$.

Proof. By using inequality (4.8) and condition (1.12), it is not hard to see that there exists a constant $C(\nu, q)$ such that

$$
\left(1+\lambda_{m, n}\right)^{\nu} \leq C(\nu, q) \frac{d(m, n)}{a_{m, n}^{q}(K) \omega_{q}}, \quad(m, n) \in A_{K}
$$

Hence,

$$
\|f\|_{\lambda, v}^{2} \leq C(\nu, q) \sum_{(m, n) \in A_{K}} \frac{d(m, n)}{a_{m, n}^{q}(K) \omega_{q}}\left\|s_{m, n}(f)\right\|_{2}^{2}=C(\nu, q)\|f\|_{K}^{2}, \quad f \in \mathcal{N}_{K} .
$$

The proof is complete.

The reader may verify that the inclusion in Theorem 4.2 becomes an equality when $a_{m, n}^{q}(K)>0, m, n \in \mathbb{Z}_{+}$, and $c_{m, n} \neq 0, m, n \in \mathbb{Z}_{+}$.

Theorem 4.3 below establishes a Sobolev-type space setting for solving (1.2). A weaker version of part (ii) was proved in [11]. The setting there was the real one. The proof presented here is simpler.

Theorem 4.3. Let $T$ be a pseudodifferential of order $v, \alpha$ a sequence satisfying (4.1), and $\mu \in \mathbb{R}$. The following assertions hold:

(i) if $\mu \geq v$, there exists a constant $C(\alpha, \mu, T)$ such that

$$
\|T(f)\|_{\alpha, \mu-\nu} \leq C(\alpha, \mu, T)\|f\|_{\lambda, \mu}, \quad f \in \mathscr{H}^{\mu}(\lambda)
$$

in particular, $T\left(\mathscr{H}^{\mu}(\lambda)\right) \subseteq \mathscr{H}^{\mu-\nu}(\alpha)$;

(ii) if $\mu \geq 0$, there exists a constant $c(\alpha, \mu, T)$ such that

$$
\|f\|_{\alpha, \mu} \leq c(\alpha, \mu, T)\|T(f)\|_{\lambda, \mu-v}, \quad f \in \mathscr{H}^{\mu}(\alpha) .
$$

Proof. (i) Let $f \in \mathscr{H}^{\alpha}(\lambda)$. Due to (4.1) and (4.8), we see that

$$
\left|c_{m, n}\right|^{2}\left(1+\alpha_{m, n}\right)^{\mu-v} \leq\left|c_{m, n}\right|^{2} C_{\alpha}^{\mu-v}\left(1+\lambda_{m, n}\right)^{\mu-v} \leq C C_{\alpha}^{\mu-v}\left(1+\lambda_{m, n}\right)^{\mu}, \quad m, n \in \mathbb{Z}_{+} .
$$


Hence,

$$
\begin{aligned}
\|T(f)\|_{\alpha, \mu-\nu}^{2} & =\sum_{m, n \in \mathbb{Z}_{+}}\left(1+\alpha_{m, n}\right)^{\mu-\nu}\left|c_{m, n}\right|^{2}\left\|s_{m, n}(f)\right\|_{2}^{2} \\
& \leq C C_{\alpha}^{\mu-v} \sum_{m, n \in \mathbb{Z}_{+}}\left(1+\lambda_{m, n}\right)^{\mu}\left\|s_{m, n}(f)\right\|_{2}^{2} .
\end{aligned}
$$

The constant $C(\alpha, \mu, T)$ in the statement (i) satisfies $C(\alpha, \mu, T)^{2}=C C_{\alpha}^{\mu-\nu}$.

(ii) It suffices to adapt the proof of (i) using the inequalities

$$
\left(1+\alpha_{m, n}\right)^{\mu} \leq C_{\alpha}^{\mu}\left(1+\lambda_{m, n}\right)^{\mu} \leq \frac{C_{\alpha}^{\mu}}{c}\left(1+\lambda_{m, n}\right)^{\mu-\nu}\left|c_{m, n}\right|^{2}
$$

instead. The constant $c(\alpha, \mu, T)$ satisfies $c c(\alpha, \mu, T)^{2}=C_{\alpha}^{\mu}$.

We close the section mentioning without proof a version of Theorem 4.3 which does not involve the condition $\mu \geq v$. The sequence $\alpha$ needs to be replaced with $\lambda$. Part (ii) holds even when $\nu$ is nonpositive.

Theorem 4.4. Let $T$ be a pseudodifferential operator of order $\nu$ and $\mu \in \mathbb{R}$. The following assertions hold:

(i) there exist a positive constant $C(T)$ such that

$$
\|T(f)\|_{\lambda, \mu-\nu} \leq C(T)\|f\|_{\lambda, \mu}, \quad f \in \mathscr{H}^{\mu}(\lambda) ;
$$

in particular, $T\left(\mathscr{H}^{\mu}(\lambda)\right) \subseteq \mathscr{H}^{\mu-\nu}(\lambda)$;

(ii) there exists a positive constant $c(T)$ such that

$$
\|f\|_{\lambda, \mu} \leq c(T)\|T(f)\|_{\lambda, \mu-\nu}, \quad f \in \mathscr{H}^{\mu}(\lambda)
$$

\section{Approximate solutions}

In this section, we describe the construction of approximate solutions of the equation $T(f)=g$. Throughout the section, $T$ will be a multiplicative operator and $K$ will be a function as described in (1.12) and obeying (1.15).

Given a Hilbert space $(\mathscr{H},[[\cdot, \cdot]])$, a closed subspace $V$, and $u \in \mathscr{H}$, we will denote by $p(u, V)$ the unique element of $V$ satisfying $[[u-p(u, V), v]]=0, v \in V$. Such an element satisfies

$$
|[[u-v]]|^{2}=|[[u-p(u, V)]]|^{2}+|[[v-p(u, V)]]|^{2}, \quad v \in V,
$$

in which $|[[\cdot]]|$ denotes the norm induced by $[[\cdot, \cdot]]$.

If $W=\left\{w_{1}, \ldots, w_{N}\right\}$ is a subset of $\Omega_{2 q}$, we define

$$
\begin{aligned}
V_{W} & :=\operatorname{span}\left\{\left(\delta_{j} \circ T^{w}\right) K(\langle\cdot, w\rangle): 1 \leq j \leq N\right\}, \\
V_{W_{T}} & :=\operatorname{span}\left\{\delta_{j}\left(K_{T}(\langle\cdot, w\rangle)\right): 1 \leq j \leq N\right\} .
\end{aligned}
$$

Due to Theorem 3.2, $V_{W}$ and $V_{W_{T}}$ are subspaces of $\mathcal{N}_{K}$ and $\mathcal{N}_{K_{T}}$, respectively. They are obviously closed. 
104 Approximations via complex spherical multiplier operators

The interpolatory properties of the spaces defined above are collected in the following result.

Theorem 5.1. Let $W=\left\{w_{1}, \ldots, w_{N}\right\}$ be a subset of $\Omega_{2 q}$ such that $\left\{\delta_{k} \circ T: k=1, \ldots, N\right\}$ is linearly independent. The following assertions hold:

(i) if $f \in \mathcal{N}_{K}$, then $s=p\left(f, V_{W}\right)$ is the unique element of $V_{W}$ satisfying

$$
\left(\delta_{j} \circ T\right)(s)=\left(\delta_{j} \circ T\right)(f), \quad j=1, \ldots, N
$$

(ii) if $g \in \mathcal{N}_{K_{T}}$, then $s=p\left(g, V_{W_{T}}\right)$ is the unique element of $V_{W_{T}}$ satisfying

$$
\delta_{j}(s)=g\left(w_{j}\right), \quad j=1, \ldots, N
$$

(iii) if $g \in \mathcal{N}_{K_{T}}, f \in \mathcal{N}_{K}$, and $g=T(f)$, then $T\left(p\left(f, V_{W}\right)\right)=p\left(g, V_{W_{T}}\right)$.

Proof. (i) Let $f \in \mathcal{N}_{K}$. Due to Corollary 2.3, we know that every $\delta_{j}$ belongs to $\mathcal{N}_{K}^{*}$, the dual of $\mathcal{N}_{K}$. Theorem 3.2 guarantees that the interpolation matrix

$$
\left(\left(\delta_{i} \circ T^{z}\right)\left(\delta_{j} \circ T^{w}\right) K(\langle z, w\rangle)\right)_{i, j=1, \ldots, N}
$$

is well defined. We now shows that it is invertible. The quadratic form $Q$ defined by the matrix in (5.5) can be written, with the help of the addition formula for spherical harmonics and of Theorem 3.3, in the form

$$
\begin{aligned}
Q & =\sum_{\mu=1}^{N} \sum_{\nu=1}^{N} c_{\mu} \bar{c}_{\nu}\left(\delta_{\mu} \circ T^{z}\right)\left(\delta_{\nu} \circ T^{w}\right) K(\langle z, w\rangle) \\
& =\sum_{\mu=1}^{N} \sum_{\nu=1}^{N} c_{\mu} \bar{c}_{\nu}\left(\delta_{\mu} \circ T^{z}\right)\left(\delta_{\nu} \circ T^{w}\right) \sum_{m, n \in \mathbb{Z}_{+}} a_{m, n}^{q}(K) P_{m, n}^{q-2}(\langle z, w\rangle) \\
& =\sum_{m, n \in \mathbb{Z}_{+}} \frac{a_{m, n}^{q}(K) \omega_{q}}{d(m, n)} \sum_{j=1}^{d(m, n)}\left|\sum_{\mu=1}^{N} c_{\mu}\left(\delta_{\mu} \circ T\right)\left(Y_{m, n}^{j}\right)\right|^{2} .
\end{aligned}
$$

Since the coefficients of this series are nonnegative, the equality $Q=0$ occurs if and only if

$$
\sum_{\mu=1}^{N} c_{\mu}\left(\delta_{\mu} \circ T\right)\left(Y_{m, n}^{j}\right)=0, \quad j=1, \ldots, d(m, n), m, n \in \mathbb{Z}_{+} .
$$

However, the fundamentality of $\left\{Y_{m, n}^{j}: j=1, \ldots, d(m, n),(m, n) \in A_{K}\right\}$ in $\mathcal{N}_{K}$ (Theorem 2.1) reveals that (5.7) is equivalent to

$$
\sum_{\mu=1}^{N} c_{\mu}\left(\delta_{\mu} \circ T\right)=0, \quad j=1, \ldots, d(m, n), m, n \in \mathbb{Z}_{+} .
$$


Since $\left\{\delta_{k} \circ T: k=1, \ldots, N\right\}$ is linearly independent, (5.8) yields $c_{\mu}=0, \mu=1, \ldots, N$. It follows that (5.3) has a unique solution, say $s_{0}$. On the other hand, by (3.7),

$$
\left\langle K(\langle\cdot, w\rangle), Y_{m, n}^{j}\right\rangle_{2}=\frac{a_{m, n}^{q}(K) \omega_{q}}{d(m, n)} \overline{Y_{m, n}^{j}(w)}, \quad w \in \Omega_{2 q}, j=1, \ldots, d(m, n), m, n \in \mathbb{Z}_{+} .
$$

Hence, by the definition of inner product in $\mathcal{N}_{K}$,

$$
\begin{aligned}
0 & =\left(\delta_{j} \circ T\right)\left(f-s_{0}\right)(w)=\left(\delta_{j} \circ T\right)\left(\sum_{(m, n) \in A_{K}} \sum_{j=1}^{d(m, n)}\left\langle f-s_{0}, Y_{m, n}^{j}\right\rangle_{2} Y_{m, n}^{j}(w)\right) \\
& =\left(\delta_{j} \circ T^{w}\right)\left(\sum_{(m, n) \in A_{K}} \frac{d(m, n)}{a_{m, n}^{q}(K) \omega_{q}} \sum_{j=1}^{d(m, n)}\left\langle f-s_{0}, Y_{m, n}^{j}\right\rangle_{2}\left\langle Y_{m, n}^{j}, K(\langle\cdot, w\rangle)\right\rangle_{2}\right) \\
& =\left(\delta_{j} \circ T^{w}\right)\left\langle f-s_{0}, K(\langle\cdot, w\rangle)\right\rangle_{K} \\
& =\left\langle f-s_{0},\left(\delta_{j} \circ T^{w}\right) K(\langle\cdot, w\rangle)\right\rangle_{K}, \quad j=1, \ldots, N .
\end{aligned}
$$

Thus, $\left\langle f-s_{0}, h\right\rangle_{K}=0, h \in V_{W}$. Therefore, by $(5.1), s_{0}=p\left(f, V_{W}\right)$. In other words, $\langle f-$ $\left.s_{0}, h\right\rangle_{K}=0, h \in V_{W}$, that is, $s_{0}=p\left(f, V_{W}\right)$.

(ii) Due to its similarity with the proof of (i), it will be omitted.

(iii) Using (i) and the hypothesis, we have that

$$
\delta_{j}\left(T\left(p\left(f, V_{W}\right)\right)\right)=\delta_{j}(T(f))=\delta_{j}(g)=g\left(w_{j}\right), \quad j=1, \ldots, N .
$$

Theorem 3.3 allows us to write

$$
T\left(p\left(f, V_{W}\right)\right)=T\left(\sum_{k=1}^{N} d_{k}\left(\delta_{\mu} \circ T^{w}\right) K(\langle\cdot, w\rangle)\right)=\sum_{k=1}^{N} d_{k} \delta_{\mu}\left(K_{T}(\langle\cdot, w\rangle)\right) \in V_{W_{T}},
$$

with $d_{k} \in \mathbb{C}, k=1, \ldots, N$. By (ii), $p\left(g, V_{W_{T}}\right)$ is the unique solution $w$ of $V_{W_{T}}$ with the property that $\delta_{j}(w)=g\left(w_{j}\right), j=1, \ldots, N$. It follows that $T\left(p\left(f, V_{W}\right)\right)=p\left(g, V_{W_{T}}\right)$.

Theorem 5.1 still holds when we substitute the linear independence hypothesis of the set $\left\{\delta_{k} \circ T: k=1, \ldots, N\right\}$ by the following pair of conditions: $c_{m, n} \neq 0,(m, n) \in A_{K}$, and $\left\{\delta_{k}: k=1, \ldots, N\right\}$ is linearly independent. The first one determines that $T$ is an isomorphism between $\mathcal{N}_{K}$ and $\mathcal{N}_{K_{T}}$.

\section{Approximations}

In this section, $T, K$, and $K_{T}$ are as in the previous section and $g$ is a fixed element in $\mathcal{N}_{K_{T}}$. We obtain error estimates for the approximation of a solution $f \in \mathcal{N}_{K}$ of (1.2) by a function of the form $p\left(f, V_{W}\right)$, for some $W$, at least in the case in which $T$ is pseudodifferential. Since $\mathcal{N}_{K}$ can be embedded in Sobolev-type spaces (Theorem 4.2), the estimates are obtained in the norm of the latter.

Corollary 2.3 shows that if $W=\left\{w_{1}, \ldots, w_{N}\right\} \subset \Omega_{2 q}$, then

$$
\mathscr{F}_{W}:=\operatorname{span}\left\{\delta_{j}: 1 \leq j \leq N\right\}
$$


is a subspace of $\mathcal{N}_{K}^{*}$. In particular, it makes sense to consider the distance between $\delta \in \mathcal{N}_{K}^{*}$ and $\mathscr{F}_{W}$ given by

$$
d^{*}\left(\delta, \mathscr{F}_{W}\right):=\inf _{\tau \in \mathscr{F}_{W}}\|\delta-\tau\|_{\mathcal{N}_{K}^{*}}
$$

in which $\|\cdot\|_{\mathcal{N}_{K}^{*}}$ denotes the usual norm of $\mathcal{N}_{K}^{*}$.

The preliminary estimates are given bellow.

Theorem 6.1. Let $f \in \mathcal{N}_{K}$ be a solution of (1.2) and $Y=\left\{w_{1}, \ldots, w_{N}\right\}$ a subset of $\Omega_{2 q}$. Assume that $\left\{\delta_{\mu} \circ T: \mu=1, \ldots, n\right\}$ is linearly independent and consider $p\left(f, V_{W}\right)$ and $p\left(g, V_{W_{T}}\right)$, as given by Theorem 5.1. If $T$ is a pseudodifferential of order $v$, then

$$
\left\|f-p\left(f, V_{W}\right)\right\|_{\lambda, v} \leq c(T) \sqrt{\sigma_{q}}\left\|g-p\left(g, V_{W_{T}}\right)\right\|_{K_{T}} \sup _{z \in \Omega_{2 q}} d^{*}\left(\delta_{z}, \mathscr{F}_{W}\right),
$$

where $c(T)$ is the constant described in Theorem 4.4 .

Proof. By Theorem 5.1, we see that

$$
\begin{aligned}
T\left(f-p\left(f, V_{W}\right)\right)(z) & =\delta_{z}\left(T(f)-T\left(p\left(f, V_{W}\right)\right)\right)=\delta_{z}\left(g-p\left(g, V_{W_{T}}\right)\right) \\
& =\left(\delta_{z}-\delta_{j}\right)\left(g-p\left(g, V_{W_{T}}\right)\right), \quad j=1, \ldots, N .
\end{aligned}
$$

Hence,

$$
\left|T\left(f-p\left(f, V_{W}\right)\right)(z)\right| \leq d^{*}\left(\delta_{z}, \mathscr{F}_{W}\right)\left\|g-p\left(g, V_{W_{T}}\right)\right\|_{K_{T}}, \quad z \in \Omega_{2 q} .
$$

Using this and Theorem 4.4, we obtain

$$
\begin{aligned}
\left\|f-p\left(f, V_{W}\right)\right\|_{\lambda, v} & \leq c(T)\left\|T\left(f-p\left(f, V_{W}\right)\right)\right\|_{\lambda, 0} \\
& =c(T)\left\|T\left(f-p\left(f, V_{W}\right)\right)\right\|_{2} \leq c(T) \sqrt{\omega_{q}}\left\|T\left(f-\left(f, V_{W}\right)\right)\right\|_{\infty} \\
& \leq c(T) \sqrt{\omega_{q}}\left\|g-p\left(g, V_{W_{T}}\right)\right\|_{K_{T}} \sup _{z \in \Omega_{2 q}} d^{*}\left(\delta_{z}, \mathscr{F}_{W}\right),
\end{aligned}
$$

concluding the proof.

Next, we will search for estimates of the two quantities on the right-hand side of the inequality in Theorem 6.1. The first one is handled in an easy manner.

Theorem 6.2. Let $W=\left\{w_{1}, \ldots, w_{N}\right\}$ be a subset of $\Omega_{2 q}$. Assume that $\left\{\delta_{k} \circ T: k=1, \ldots, n\right\}$ is linearly independent and consider $p\left(g, V_{W_{T}}\right)$, as given by Theorem 5.1(ii). Then the following inequalities hold:

(i) $\left\|g-p\left(g, V_{W_{T}}\right)\right\|_{K_{T}} \leq\|g\|_{K_{T}}, g \in \mathcal{N}_{K_{T}}$;

(ii) $\left\|g-p\left(g, V_{W_{T}}\right)\right\|_{K_{T}} \leq \sqrt{\omega_{q}}\|g\|_{K_{T} * K_{T}} \sup _{z \in \Omega_{2 q}} d^{*}\left(\delta_{z}, \mathscr{F}_{W}\right), g \in \mathcal{N}_{K_{T} * K_{T}}$. 
Proof. The first assertion of the theorem is consequence of (5.1). As for the second, if $g \in \mathcal{N}_{K_{T} * K_{T}}$, we use the Cauchy-Schwarz inequality to obtain

$$
\begin{aligned}
\left\|g-p\left(g, V_{W_{T}}\right)\right\|_{K_{T}}^{2}= & \left\langle g, g-p\left(g, V_{W_{T}}\right)\right\rangle_{K_{T}} \\
= & \frac{1}{\omega_{q}} \sum_{(m, n) \in A_{K_{T}}} \frac{d(m, n)}{a_{m, n}^{q}(K)\left|c_{m, n}\right|^{2}} \sum_{j=1}^{d(m, n)}\left\langle g, Y_{m, n}^{j}\right\rangle_{2}\left\langle Y_{m, n}^{j}, g-p\left(g, V_{W_{T}}\right)\right\rangle_{2} \\
\leq & \left(\frac{1}{\omega_{q}^{2}} \sum_{(m, n) \in A_{K_{T}}} \frac{d(m, n)^{2}}{\left(a_{m, n}^{q}(K)\right)^{2}\left|c_{m, n}\right|^{4}} \sum_{j=1}^{d(m, n)}\left|\left\langle g, Y_{m, n}^{j}\right\rangle_{2}\right|^{2}\right)^{1 / 2} \\
& \times\left(\sum_{m, n \in \mathbb{Z}_{+}} \sum_{j=1}^{d(m, n)}\left|\left\langle g-p\left(g, V_{W_{T}}\right), Y_{m, n}^{j}\right\rangle_{2}\right|^{2}\right)^{1 / 2} \\
= & \|g\|_{K_{T} * K_{T}}\left\|g-p\left(g, V_{W_{T}}\right)\right\|_{2}, \quad g \in \mathcal{N}_{K_{T} * K_{T}} .
\end{aligned}
$$

The procedure used in the proof of Theorem 6.1 yields

$$
\begin{aligned}
\left\|g-p\left(g, V_{W_{T}}\right)\right\|_{2} & \leq \sqrt{\omega_{q}}\left\|g-p\left(g, V_{W_{T}}\right)\right\|_{\infty} \\
& \leq \sqrt{\omega_{q}}\left\|g-p\left(g, V_{W_{T}}\right)\right\|_{K_{T}} \sup _{z \in \Omega_{2 q}} d^{*}\left(\delta_{z}, \mathscr{F}_{W}\right) .
\end{aligned}
$$

Thus, the inequality follows in the case $\left\|g-p\left(g, V_{W_{T}}\right)\right\|_{K_{T}} \neq 0$. Otherwise, it is trivial.

The bounding of the second quantity in the inequality of Theorem 6.1 requires a more intricate argument. We adapt a procedure introduced in [3]. However, a more sophisticated argument involving positive quadratures may be found in [10]. To do that, we need to deal with the mesh norm of a subset $W$ of $\Omega_{2 q}$, that is, the positive number given by

$$
\eta=\eta(W):=\sup _{z \in \Omega_{2 q}}\left\{\inf _{w \in W} d_{2 q}\left(\Upsilon_{q}(z), \Upsilon_{q}(w)\right)\right\}
$$

in which $d_{2 q}$ denotes the usual geodesic distance on $S^{2 q-1}$ and $\Upsilon_{q}$ stands for the bijective identification $\Upsilon_{q}: \Omega_{2 q} \rightarrow S^{2 q-1}$ given by

$$
\Upsilon_{q}\left(x_{1}+i y_{1}, \ldots, x_{q}+i y_{q}\right)=\left(x_{1}, y_{1}, \ldots, x_{q}, y_{q}\right) .
$$

In what follows, we will deal with functions in $\mathscr{C}\left(\Omega_{2 q}\right)$ and their restrictions to a finite subset $W$ of $\Omega_{2 q}$. The uniform norm of $\mathscr{C}\left(\Omega_{2 q}\right)$ will be denoted by $\|\cdot\|_{\infty}$ while the uniform norm of $\mathscr{C}(W)$ will be written as $\|\cdot\|_{W}$. We will not distinguish between the functions and their restrictions.

Proposition 6.3. Let $W$ be a subset of $\Omega_{2 q}$. If $\kappa$ is a positive integer at most $(2 \eta)^{-1}$, then

$$
\|g\|_{W} \leq\|g\|_{\infty} \leq 2\|g\|_{W}, \quad g \in \bigoplus_{k+l=0}^{\kappa} \mathscr{H}_{k, l}\left(\Omega_{2 q}\right) .
$$


108 Approximations via complex spherical multiplier operators

Proof. The first inequality is obvious. As for the other, let $g \in \oplus_{k+l=0}^{\kappa} \mathscr{H}_{k, l}\left(\Omega_{2 q}\right)$. We can assume that $\|g\|_{\infty} \neq 0$ since otherwise there is nothing to be proved. Pick $\zeta \in \Omega_{2 q}$ such that $\|g\|_{\infty}=|g(\zeta)|$. Using (6.9), we can select a $w_{0} \in W$ such that $d_{2 q}\left(\Upsilon_{q}(\zeta), \Upsilon_{q}\left(w_{0}\right)\right) \leq$ $\eta \leq 1 / 2 \kappa$. Using the well-known Markov inequality, we deduce that

$$
\left|\frac{g(\zeta)}{\|g\|_{\infty}}-\frac{g\left(w_{0}\right)}{\|g\|_{\infty}}\right| \leq \kappa d_{2 q}\left(\Upsilon_{q}(\zeta), \Upsilon_{q}\left(w_{0}\right)\right) \leq \frac{1}{2}
$$

It follows that

$$
\left|\frac{g\left(w_{0}\right)}{\|g\|_{\infty}}\right| \geq\left|\frac{g(\zeta)}{\|g\|_{\infty}}\right|-\left|\frac{g(\zeta)}{\|g\|_{\infty}}-\frac{g\left(w_{0}\right)}{\|g\|_{\infty}}\right| \geq \frac{1}{2} .
$$

Consequently,

$$
\frac{1}{\|g\|_{\infty}} \sup _{w \in W}|g(w)| \geq \frac{\left|g\left(w_{0}\right)\right|}{\|g\|_{\infty}} \geq \frac{1}{2} .
$$

Thus, $\|g\|_{\infty} \leq 2\|g\|_{W}$, completing the proof of the proposition.

Corollary 6.4. Let $W$ be a subset of $\Omega_{2 q}$. If $\kappa$ is a positive integer at most $(2 \eta)^{-1}$, then the map

$$
\left.h \in \bigoplus_{k+l=0}^{\kappa} \mathcal{H}_{k, l}\left(\Omega_{2 q}\right) \stackrel{\psi}{\longmapsto} h\right|_{W} \in \mathscr{C}(W)
$$

is a monomorphism.

Lemma 6.5 is purely technical and probably well known. We use $\left\{\varepsilon_{1}, \ldots, \varepsilon_{N}\right\}$ to denote the canonical basis for $\mathbb{C}^{N}$ while $\left\{\varepsilon_{1}^{*}, \ldots, \varepsilon_{N}^{*}\right\}$ denotes the corresponding dual basis.

Lemma 6.5. Let $W=\left\{w_{1}, \ldots, w_{N}\right\}$ be a subset of $\Omega_{2 q}$. Then the dual space of $\mathscr{C}(W)$ coincides with $\operatorname{span}\left\{\left.\delta_{j}\right|_{\mathscr{C}(W)}: j=1, \ldots, N\right\}$.

Proof. The space $\mathbb{C}^{N}$ endowed with its maximum norm is isomorphic to $\left(\mathscr{C}(W),\|\cdot\|_{\infty}\right)$ and the isomorphism is given by

$$
S(g)=\left(g\left(w_{1}\right), \ldots, g\left(w_{N}\right)\right), \quad g \in \mathscr{C}(W) .
$$

In particular, the adjoint $S^{*}$ of $S$ defines an isomorphism between $\left(\mathbb{C}^{N}\right)^{*}$ and $\mathscr{C}(W)^{*}$. If $\Lambda \in \mathscr{C}(W)^{*}$, then $S^{*}(\Gamma)=\Lambda$ for some $\Gamma$ in $\left(\mathbb{C}^{N}\right)^{*}$ given by

$$
\Gamma=\sum_{j=1}^{N} a_{j} \varepsilon_{j}^{*}, \quad a_{1}, \ldots, a_{N} \in \mathbb{C} .
$$


Now, by using the definition of $S^{*}$ and direct computation, we obtain

$$
\Lambda(g)=\left(\sum_{j=1}^{N} a_{j} \delta_{j}\right)(g), \quad g \in \mathscr{C}(W)
$$

Hence, $\Lambda=\sum_{j=1}^{N} a_{j} \delta_{j}$ is an element of $\operatorname{span}\left\{\left.\delta_{j}\right|_{\mathscr{C}(W)}: j=1, \ldots, N\right\}$. On the other hand, if $\Gamma=\sum_{j=1}^{N} b_{j} \delta_{j}, b_{1}, \ldots, b_{N} \in \mathbb{C}$, then the functional $\Lambda$ of $\left(\mathbb{C}^{N}\right)^{*}$ given by $\Lambda=\sum_{j=1}^{N} b_{j} \varepsilon_{j}^{*}$ satisfies $\Gamma=S^{*}(\Lambda)$. Thus, $\Gamma \in \mathscr{C}(W)^{*}$.

THeOREM 6.6. Let $W$ be a finite subset of cardinality $N$ of $\Omega_{2 q}$. If $\kappa$ is a positive integer at most $(2 \eta)^{-1}$ and $z \in \Omega_{2 q}$, then $\delta_{z}$ can be written as

$$
\delta_{z}(g)=\sum_{\mu=1}^{N} c_{\mu} \delta_{\mu}(g), \quad g \in \bigoplus_{k+l=0}^{\kappa} \mathcal{H}_{k, l}\left(\Omega_{2 q}\right)
$$

in which the complex numbers $c_{1}, \ldots, c_{N}$ satisfy $\sum_{\mu=1}^{N}\left|c_{\mu}\right| \leq 2$.

Proof. The map $\psi$ in Corollary 6.4 is an isomorphism between spaces $\oplus_{k+l=0}^{\kappa} \mathscr{H}_{k, l}\left(\Omega_{2 q}\right)$ and $\psi\left(\oplus_{k+l=0}^{\kappa} \mathscr{H}_{k, l}\left(\Omega_{2 q}\right)\right)$. Due to Proposition 6.3,

$$
\left\|\psi^{-1}\right\|=\sup _{\|\psi(g)\|_{W} \leq 1}\left\|\psi^{-1}(\psi(g))\right\|_{\infty}=\sup _{\|g\|_{W} \leq 1}\|g\|_{\infty} \leq 2, \quad g \in \bigoplus_{k+l=0}^{\kappa} \mathscr{H}_{k, l}\left(\Omega_{2 q}\right) .
$$

It follows that

$$
\psi^{*}: \psi\left(\oplus_{k+l=0}^{\kappa} \mathscr{H}_{k, l}\left(\Omega_{2 q}\right)\right)^{*} \longmapsto\left(\oplus_{k+l=0}^{\kappa} \mathcal{H}_{k, l}\left(\Omega_{2 q}\right)\right)^{*}
$$

is an isomorphism and $\left\|\psi^{*-1}\right\|=\left\|\psi^{-1}\right\| \leq 2$. It is now clear that there is a $\Lambda$ in the space $\psi\left(\oplus_{k+l=0}^{\kappa} \mathscr{H}_{k, l}\left(\Omega_{2 q}\right)\right)^{*}$ such that $\psi^{*}(\Lambda)$ coincides with the restriction of $\delta_{z}$ to $\oplus_{k+l=0}^{\kappa} \mathscr{H}_{k, l}\left(\Omega_{2 q}\right)$. Also,

$$
\|\Lambda\|=\left\|\psi^{*-1}\left(\delta_{z}\right)\right\| \leq\left\|\psi^{*-1}\right\| \leq 2
$$

By the Hahn-Banach theorem, there exists $\vartheta \in \mathscr{C}(W)^{*}$ such that $\|\vartheta\|=\|\Lambda\|$ and

$$
\vartheta(\psi(g))=\Lambda(\psi(g)), \quad g \in \bigoplus_{k+l=0}^{\kappa} \mathscr{H}_{k, l}\left(\Omega_{2 q}\right)
$$

Next, we use Lemma 6.5 to select complex numbers $c_{1}, \ldots, c_{N}$ such that

$$
\vartheta(\psi(g))=\sum_{j=1}^{N} c_{j} \delta_{j}(\psi(g)), \quad g \in \bigoplus_{k+l=0}^{\kappa} \mathcal{H}_{k, l}\left(\Omega_{2 q}\right) .
$$


110 Approximations via complex spherical multiplier operators

We observe that $\sum_{j=1}^{N}\left|c_{j}\right|=\|\Lambda\|=\|9\| \leq 2$. Finally, noticing that

$$
\delta_{j}(\psi(g))=\delta_{j}(g), \quad g \in \oplus_{k+l=0}^{\kappa} \mathscr{H}_{k, l}\left(\Omega_{2 q}\right)
$$

and using (6.22), (6.23), and (6.24), we obtain

$$
\delta_{z}(g)=\Lambda(\psi(g))=\sum_{j=1}^{N} c_{j} \delta_{j}(\psi(g))=\left(\sum_{j=1}^{N} c_{j} \delta_{j}\right)(g), \quad g \in \bigoplus_{k+l=0}^{\kappa} \mathscr{H}_{k, l}\left(\Omega_{2 q}\right)
$$

This completes the proof.

The dual distance (6.2) has the estimate given below.

Theorem 6.7. Let $W=\left\{w_{1}, \ldots, w_{N}\right\}$ be a subset of $\Omega_{2 q}$ and $z \in \Omega_{2 q}$. If $\kappa$ is a positive integer at most $(2 \eta)^{-1}$, then

$$
d^{*}\left(\delta_{z}, \mathscr{F}_{W}\right) \leq 3\left(\sum_{m+n>\kappa} a_{m, n}^{q}(K)\left|c_{m, n}\right|^{2}\right)^{1 / 2}
$$

Proof. We take $\delta_{z}$ as in Theorem 6.6 and define $c_{0}=-1, w_{0}=z$ and the linear functional

$$
v:=-\delta_{z}+\sum_{\mu=1}^{N} c_{\mu} \delta_{\mu}=\sum_{\mu=0}^{N} c_{\mu} \delta_{\mu}
$$

Corollary 2.3 guarantees that $v \in \mathcal{N}_{K_{T}}^{*}$. The expansion of $g$ as in (1.8) guarantees that

$$
v(g)=\sum_{m+n>\kappa} v\left(s_{m, n}(g)\right), \quad g \in \mathcal{N}_{K_{T}}
$$

Defining

$$
\sum_{m+n>\kappa}\left|s_{m, n}(g)\left(w_{M}\right)\right|:=\max \left\{\sum_{m+n>\kappa}\left|s_{m, n}(g)\left(w_{k}\right)\right|: k=0, \ldots, N\right\}, \quad g \in \mathcal{N}_{K_{T}},
$$

and using the fact that $\sum_{k=1}^{N}\left|c_{k}\right| \leq 2$, we see that

$$
\begin{aligned}
|v(g)| & \leq \sum_{m+n>\kappa} \sum_{k=0}^{N}\left|c_{k}\right|\left|s_{m, n}(g)\left(w_{k}\right)\right|=\sum_{k=0}^{N}\left|c_{k}\right|\left(\sum_{m+n>\kappa}\left|s_{m, n}(g)\left(w_{k}\right)\right|\right) \\
& \leq \sum_{k=0}^{N}\left|c_{k}\right|\left(\sum_{m+n>\kappa}\left|s_{m, n}(g)\left(w_{M}\right)\right|\right) \leq 3 \sum_{m+n>\kappa}\left|s_{m, n}(g)\left(w_{M}\right)\right|, \quad g \in \mathcal{N}_{K_{T}} .
\end{aligned}
$$


Finally, the Cauchy-Schwartz inequality and the addition formula imply that

$$
\begin{aligned}
d^{*}\left(\delta_{z}, \mathscr{F}_{W}\right) & \leq\left\|\delta_{z}-\sum_{k=1}^{N} c_{k} \delta_{k}\right\|_{\mathcal{N}_{K_{T}}^{*}} \\
& =\sup _{\|h\|_{K_{T}} \leq 1}|v(h)| \leq 3 \sup _{\|h\|_{K_{T}} \leq 1}\left\{\sum_{m+n>\kappa}\left|s_{m, n}(h)\left(w_{M}\right)\right|\right\} \\
& =3 \sup _{\|h\|_{K_{T}} \leq 1}\left\{\sum_{\substack{m+n>\mathcal{K} \\
(m, n) \in A_{K_{T}}}}\left|s_{m, n}(h)\left(w_{M}\right)\right|\right\} \\
& \leq 3 \sup _{\|h\|_{K_{T}} \leq 1}\|h\|_{K_{T}}\left(\sum_{\substack{m+n>\kappa \\
(m, n) \in A_{K_{T}}}} \frac{a_{m, n}^{q}(K)\left|c_{m, n}\right|^{2} \omega_{q}}{d(m, n)} \sum_{j=1}^{d(m, n)}\left|Y_{m, n}^{j}\left(w_{M}\right)\right|^{2}\right)^{1 / 2} \\
& \leq 3\left(\sum_{m+n>\kappa} a_{m, n}^{q}(K)\left|c_{m, n}\right|^{2}\right)^{1 / 2},
\end{aligned}
$$

completing the proof.

\section{Error estimates in specific cases}

It is now evident that it is not possible to further estimate $d^{*}\left(\delta_{z}, \mathscr{F}_{W}\right)$, unless additional assumptions on either $T$ or $K$ are made. In this section, we present estimates in the case when $T$ is pseudodifferential and the sequence $\left\{a_{m, n}^{q}(K)\right\}_{m, n \in \mathbb{Z}_{+}}$obeys a stronger decay condition. Throughout the section, $T$ and $K$ are as described at the beginning of Section 5 and $W$ is a finite subset of $\Omega_{2 q}$ with mesh norm $\eta$.

Theorem 7.1. Let $\kappa$ be a positive integer such that $(2 \eta)^{-1} \in(\kappa, \kappa+1]$. Assume that $T$ is a pseudodifferential of order $v$ and that $a_{m, n}^{q}(K) \leq D\left(1+\lambda_{m, n}\right)^{-s-1}, m+n>\kappa$, for some $s>v$ and some $D>0$. Then, there exists a positive constant $C_{1}=C_{1}(s-v)$ such that

$$
\sum_{m+n>\kappa} a_{m, n}^{q}(K)\left|c_{m, n}\right|^{2} \leq C_{1} \eta^{2(s-\nu)}
$$

Proof. Using the hypotheses and borrowing the notation from (4.7) and (4.8), we have that

$$
\begin{aligned}
a_{m, n}^{q}( & K)\left|c_{m, n}\right|^{2} \\
& \leq C D\left(1+\lambda_{m, n}\right)^{\nu-s-1} \\
& \leq C D K(1+m+n)^{2 \nu-2 s-2}, \quad m+n>\kappa .
\end{aligned}
$$


112 Approximations via complex spherical multiplier operators

Since $2 v-2 s<0$,

$$
\begin{aligned}
\sum_{m+n>\kappa} a_{m, n}^{q}(K)\left|c_{m, n}\right|^{2} & \leq \sum_{m+n>\kappa} \operatorname{CDK}(1+m+n)^{2 v-2 s-2} \\
& =C D K \sum_{j=1}^{\infty}\left(\sum_{m+n=\kappa+j}(1+m+n)^{2 v-2 s-2}\right) \\
& =C D K \sum_{j=1}^{\infty}(1+\kappa+j)^{2 \nu-2 s-1} \leq C D K \int_{1}^{\infty}(1+\kappa+t)^{2 \nu-2 s-1} d t \\
& =\frac{C D K}{2 s-2 v}(2+\kappa)^{2 v-2 s} \leq \frac{C D K 2^{2 s-2 v}}{2 s-2 v} \eta^{2 s-2 v},
\end{aligned}
$$

which is the desired inequality.

Theorem 7.2. Let $\kappa$ be a positive integer such that $(2 \eta)^{-1} \in(\kappa, \kappa+1]$. Assume that $T$ is a pseudodifferential of order $v$ and that $a_{m, n}^{q}(K) \leq D \exp \left(-a(1+m+n)^{s}\right), m+n>\kappa$, for some $s>0$ and some positive constants $a$ and $D$. Then there exists a positive constant $C_{2}=C_{2}(s, a)$ such that

$$
\sum_{m+n>\kappa} a_{m, n}^{q}(K)\left|c_{m, n}\right|^{2} \leq \begin{cases}C_{2} e^{-a(2 \eta)^{-s}}, & 2 \nu+2 \leq s, \\ C_{2}\left(\frac{4 \eta+1}{2 \eta}\right)^{2 \nu-s+1} e^{-a(2 \eta)^{-s}}, & s<2 \nu+2 \leq s(a+1) .\end{cases}
$$

Proof. The procedure employed at the beginning of the proof of Theorem 7.1 produces the inequality

$$
a_{m, n}^{q}(K)\left|c_{m, n}\right|^{2} \leq C D K e^{-a(1+m+n)^{s}}(1+m+n)^{2 \nu}, \quad m+n>\kappa .
$$

If $2 v-s+2 \leq 0$, then

$$
\begin{aligned}
\sum_{m+n>\kappa} a_{m, n}^{q}(K)\left|c_{m, n}\right|^{2} & \leq C D K \sum_{m+n>\kappa} e^{-a(1+m+n)^{s}}(1+m+n)^{s-2} \\
& \leq C D K \int_{1}^{\infty} e^{-a(1+\kappa+t)^{s}}(1+\kappa+t)^{s-1} d t \\
& =\frac{C D K}{a s} e^{-a(2+\kappa)^{s}} \leq \frac{C D K}{a s} e^{-a(2 \eta)^{-s}} .
\end{aligned}
$$

Thus, we assume that $2 v-s+2>0$. Certainly,

$$
\sum_{m+n>\kappa} a_{m, n}^{q}(K)\left|c_{m, n}\right|^{2} \leq C D K \int_{1}^{\infty}(1+\kappa+t)^{2 v+1} e^{-a(1+\kappa+t)^{s}} d t
$$


and we have to handle the integral $I$ in the left-hand side of the inequality. Integrating by parts we obtain

$$
\begin{aligned}
I & =\int_{1}^{\infty}(1+\kappa+t)^{2 \nu-s+2}(1+\kappa+t)^{s-1} e^{-a(1+\kappa+t)^{s}} d t \\
& =\frac{(2+\kappa)^{2 \nu-s+2}}{a s e^{a(2+\kappa)^{s}}}+\frac{2 \nu-s+2}{a s} \int_{1}^{\infty}(1+\kappa+t)^{2 \nu-s+1} e^{-a(1+\kappa+t)^{s}} d t \\
& \leq \frac{(2+\kappa)^{2 \nu-s+2}}{a s e^{a(2+\kappa)^{s}}}+\frac{2 \nu-s+2}{a s(2+\kappa)^{s}} \int_{1}^{\infty}(1+\kappa+t)^{2 v+1} e^{-a(1+t+\kappa)^{s}} d t \\
& =\frac{(2+\kappa)^{2 \nu-s+2}}{a s e^{a(2+\kappa)^{s}}}+\frac{2 \nu-s+2}{a s(2+\kappa)^{s}} I .
\end{aligned}
$$

In particular,

$$
I \leq \frac{(2+\kappa)^{2 v-s+2}}{s a e^{a(2+\kappa)^{s}}}+\frac{2 v-s+2}{s a} I .
$$

If $s(a+1)>2 v+2$, we further estimate to reach

$$
I \leq D^{\prime} \frac{(2+\kappa)^{2 \nu-s+2}}{a s e^{a(2+\kappa)^{s}}} \leq \frac{D^{\prime}}{a s}\left(2+(2 \eta)^{-1}\right)^{2 v-s+1} e^{-a(2 \eta)^{-s}}
$$

where $D^{\prime}=s a /(s(a+1)-2 v-2)>0$. If $s(a+1)=2 v+2$, the estimate above occurs with $D^{\prime}=2^{s} /\left(2^{s}-1\right)$. This completes the proof of the theorem.

Usually one seeks estimates when the mesh norm is close to zero, that is, when the set $Y$ is somehow dense on the sphere. If such assumption is added to the hypotheses, the following version of Theorem 7.2 holds.

Theorem 7.3. Let $\kappa$ be a positive integer such that $(2 \eta)^{-1} \in(\kappa, \kappa+1]$. Assume that $T$ is a pseudodifferential of order $v$ and that $a_{m, n}^{q}(K) \leq D \exp \left(-a(1+m+n)^{s}\right), m+n>\kappa$, for some $s \in(0,2 v+2)$ and some positive constants $a$ and $D$. If

$$
\kappa>-2+\left(\frac{2 v-s+2}{s a}\right)^{1 / s}
$$

then there exists a positive constant $C_{2}$ such that

$$
\sum_{m+n>\kappa} a_{m, n}^{q}(K)\left|c_{m, n}\right|^{2} \leq C_{2}\left(\frac{4 \eta+1}{2 \eta}\right)^{2 v-s+2} e^{-a /(2 \eta)^{s}} .
$$

Proof. It is very easy to see that if $\kappa$ satisfies (7.11), then $(2 \nu-s+2) s^{-1} a^{-1}(2+\kappa)^{-s}<1$. The second half of the proof of Theorem 7.2 now implies that

$$
I \leq C_{2} \frac{(2+\kappa)^{2 v-s+2}}{s a e^{(2+\kappa)^{s} a}}
$$

in which $C_{2}$ is independent of $s, a$, and $\kappa$. The rest follows. 
114 Approximations via complex spherical multiplier operators

The following estimates follow from the results in this and the previous section. They encompass those in [11] and consider several other situations including that when $g \in$ $\mathcal{N}_{K_{T}}$.

Theorem 7.4. Let $\kappa$ be a positive integer such that $(2 \eta)^{-1} \in(\kappa, \kappa+1]$. Assume the notation and hypotheses of Theorem 7.1. The following assertions hold.

(i) If $a_{m, n}^{q}(K) \leq D\left(1+\lambda_{m, n}\right)^{-s-1}, m+n>\kappa$, for some $s>v$ and some $D>0$, then

$$
\left\|f-p\left(f, V_{W}\right)\right\|_{\lambda, v} \leq 3 c(T) \sqrt{C_{1} \omega_{q}} \eta^{s-\nu}\|g\|_{K_{T}}
$$

If $g \in \mathcal{N}_{K_{T} * K_{T}}$, then the inequality above specializes to

$$
\left\|f-p\left(f, V_{W}\right)\right\|_{\lambda, v} \leq 9 c(T) C_{1} \omega_{q} \eta^{2(s-v)}\|g\|_{K_{T} * K_{T}} .
$$

(ii) If $a_{m, n}^{q}(K) \leq D \exp \left(-a(1+m+n)^{s}\right), m+n>\kappa$, for some $s>0$ and some positive constants $a$ and $D$, then

$$
\begin{aligned}
& \left\|f-p\left(f, V_{W}\right)\right\|_{\lambda, v} \\
& \leq \begin{cases}3 c(T) \sqrt{C_{2} \omega_{q}} e^{-a / 2(2 \eta)^{s}}\|g\|_{K_{T}}, & 2 v+2 \leq s, \\
3 c(T) \sqrt{C_{2} \omega_{q}}\left(\frac{4 \eta+1}{2 \eta}\right)^{\nu-(s-1) / 2} e^{-a / 2(2 \eta)^{s}}\|g\|_{K_{T}}, & s<2 v+2 \leq s(a+1) .\end{cases}
\end{aligned}
$$

If $g \in \mathcal{N}_{K_{T} * K_{T}}$, the inequality specializes to

$$
\begin{aligned}
& \left\|f-p\left(f, V_{W}\right)\right\|_{\lambda, v} \\
& \leq \begin{cases}9 C(T) C_{2} \omega_{q} e^{-a /(2 \eta)^{s}}\|g\|_{K_{T} * K_{T}}, & 2 v+2 \leq s, \\
9 C(T) C_{2} \omega_{q}\left(\frac{4 \eta+1}{2 \eta}\right)^{2 \nu-s+1} e^{-a /(2 \eta)^{s}}\|g\|_{K_{T} * K_{T}}, & s<2 v+2 \leq s(a+1) .\end{cases}
\end{aligned}
$$

(iii) If $a_{m, n}^{q}(K) \leq D \exp \left(-a(1+m+n)^{s}\right), m+n>\kappa$, for some $s \in(0,2 v+1)$ and some positive constants $a$ and $D$, and in addition,

$$
\kappa \geq-2+\left(\frac{2 v-s+2}{s a}\right)^{1 / s}
$$

then

$$
\left\|f-p\left(f, V_{W}\right)\right\|_{\lambda, v} \leq 3 c(T) \sqrt{C_{2} \omega_{q}}\left(\frac{4 \eta+1}{2 \eta}\right)^{\nu+1-s / 2} e^{-a / 2(2 \eta)^{s}}\|g\|_{K_{T}} .
$$

If $g \in \mathcal{N}_{K_{T} * K_{T}}$, then this inequality specializes to

$$
\left\|f-p\left(f, V_{W}\right)\right\|_{\lambda, v} \leq 9 c(T) C_{2} \omega_{q}\left(\frac{4 \eta+1}{2 \eta}\right)^{2 v-s+2} e^{-a /(2 \eta)^{s}}\|g\|_{K_{T} * K_{T}} .
$$




\section{Acknowledgment}

This work was partially supported by CAPES-Brazil.

\section{References}

[1] J. N. Boyd and P. N. Raychowdhury, Zonal harmonic functions from two-dimensional analogs of Jacobi polynomials, Applicable Anal. 16 (1983), no. 3, 243-259.

[2] G. B. Folland, Real Analysis. Modern Techniques and Their Applications, 2nd ed., Pure and Applied Mathematics, John Wiley \& Sons, New York, 1999.

[3] K. Jetter, J. Stöckler, and J. D. Ward, Error estimates for scattered data interpolation on spheres, Math. Comp. 68 (1999), no. 226, 733-747.

[4] T. H. Koornwinder, The addition formula for Jacobi polynomials. II: The Laplace type integral representation and the product formula, Tech. Report TW 133, Math. Centrum, Afd. Toegepaste Wisk., Amsterdam, 1972.

[5] Z. Luo and J. Levesley, Error estimates and convergence rates for variational Hermite interpolation, J. Approx. Theory 95 (1998), no. 2, 264-279.

[6] W. R. Madych and S. A. Nelson, Multivariate interpolation and conditionally positive definite functions, Approx. Theory Appl. 4 (1988), no. 4, 77-89.

[7] Multivariate interpolation and conditionally positive definite functions. II, Math. Comp. 54 (1990), no. 189, 211-230.

[8] V. A. Menegatto and C. P. Oliveira, Annihilating properties of convolution operators on complex spheres, to appear in Anal. Math.

[9] V. A. Menegatto and A. P. Peron, Strict positive definiteness on spheres via disk polynomials, Int. J. Math. Math. Sci. 31 (2002), no. 12, 715-724.

[10] H. N. Mhaskar, F. J. Narcowich, and J. D. Ward, Spherical Marcinkiewicz-Zygmund inequalities and positive quadrature, Math. Comp. 70 (2001), no. 235, 1113-1130.

[11] T. M. Morton and M. Neamtu, Error bounds for solving pseudodifferential equations on spheres by collocation with zonal kernels, J. Approx. Theory 114 (2002), no. 2, 242-268.

C. P. Oliveira: Instituto de Ciências Exatas, Universidade Federal de Itajubá, Caixa Postal 50, 37500903 Itajubá, MG, Brazil

E-mail address: oliveira@unifei.edu.br 


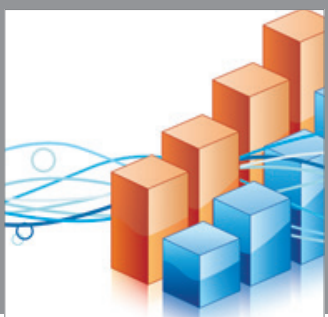

Advances in

Operations Research

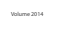

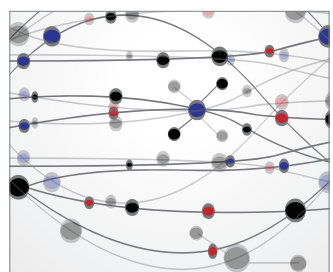

\section{The Scientific} World Journal
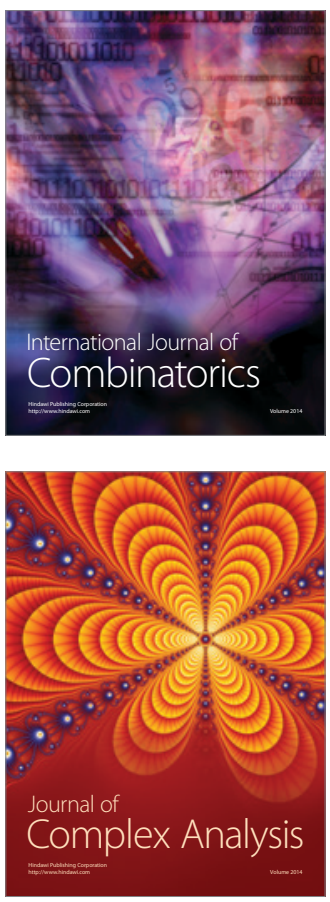

International Journal of

Mathematics and

Mathematical

Sciences
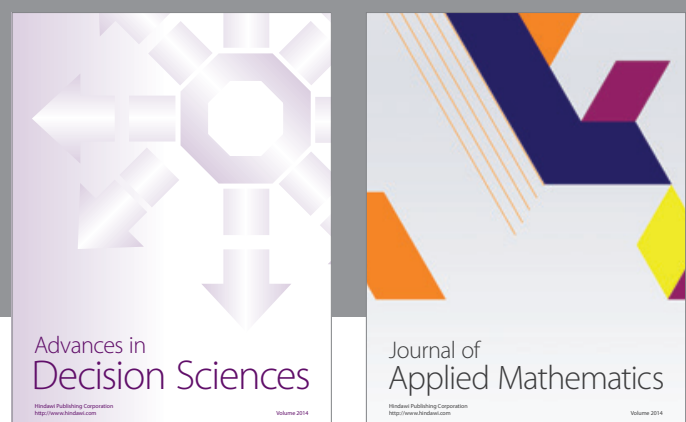

Journal of

Applied Mathematics
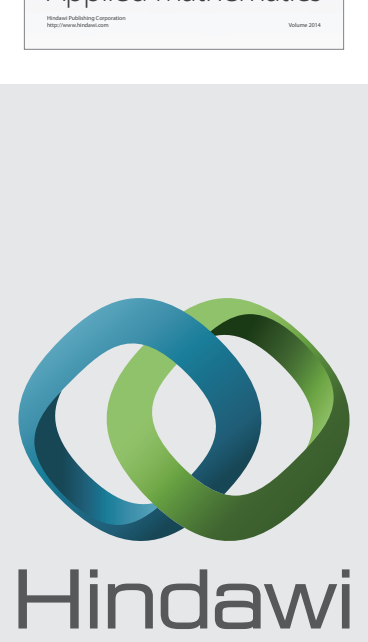

Submit your manuscripts at http://www.hindawi.com
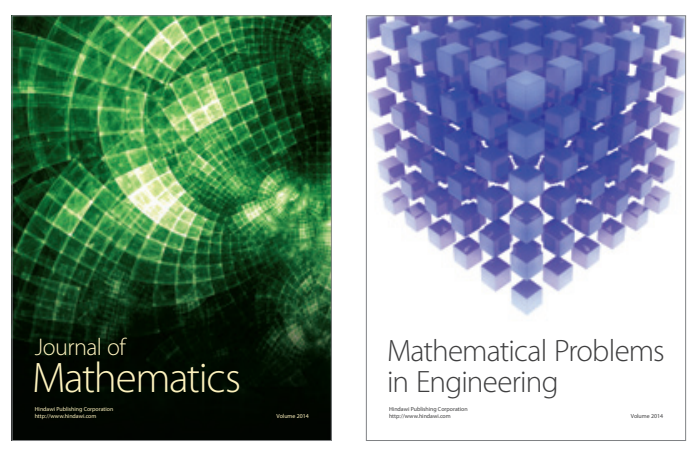

Mathematical Problems in Engineering
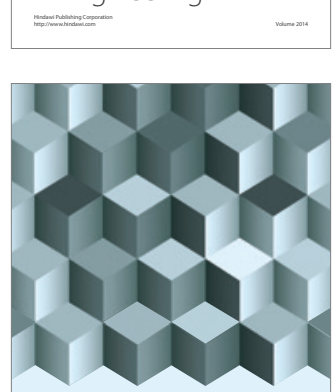

Journal of

Function Spaces
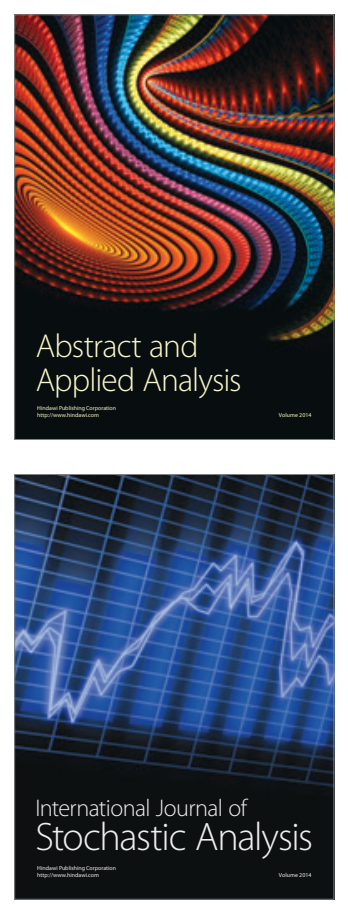

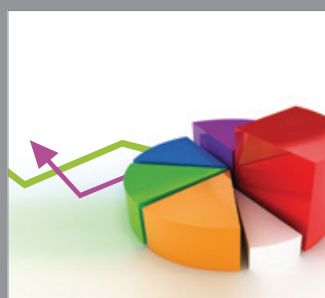

ournal of

Probability and Statistics

Promensencen
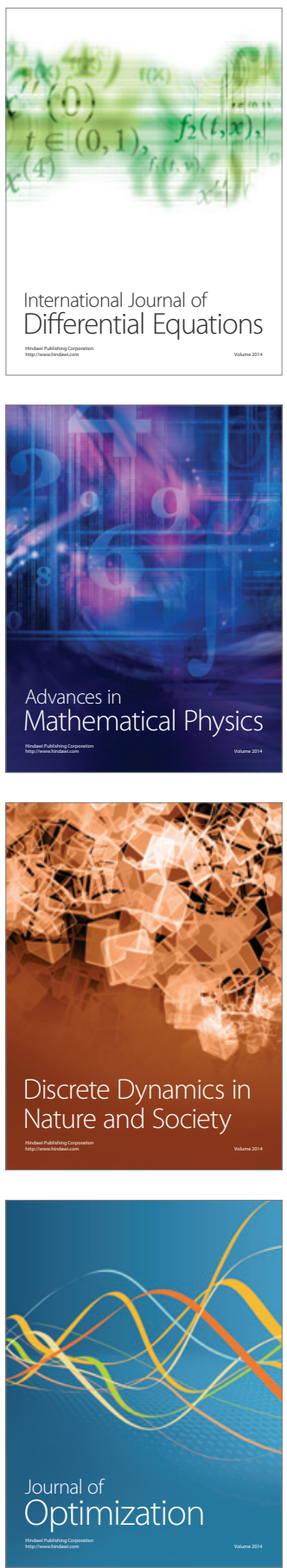